\section{Distância percebida até as instalações de lazer e sua associação com a prática de atividade física e de exercícios em adolescentes de Curitiba, Paraná, Brasil}

\author{
Perceived distance to recreational facilities and \\ the association with physical activity and exercise \\ among adolescents in Curitiba, Paraná State, Brazil
}

La percepción de la distancia a las instalaciones de ocio y su relación con la actividad física y el ejercicio en los adolescentes de Curitiba, Paraná, Brasil

1 Pontifícia Universidade
Católica do Paraná, Curitiba,
Brasil.
2 Universidade Federal do
Paraná, Curitiba, Brasil.
3 Universidade Tecnológica
Federal do Paraná, Curitiba,
Brasil.
Correspondence
A. V. Lima
Pontifícia Universidade
Católica do Paraná.
Rua Imaculada Conceição
1155, Curitiba, PR 80215-901,
Brasil.
alexvieira@hotmail.com

Abstract

The aim of this study was to verify the association between perceived distance to recreational facilities (parks, gyms, sports courts, and bike lanes) for physical activity and exercise among adolescents in Curitiba, Paraná State, Brazil. A cross-sectional study was conducted with a representative sample of 1,474 adolescents (14-18 years) enrolled in public schools. There was an inverse association between distance $(\geq 31 \mathrm{~min}$ utes) to gyms and sports courts and physical activity among boys ( $P R=0.78$; 95\%CI: 0.69-0.88 and $P R=0.79$; 95\%CI: 0.66-0.95, respectively). The number of facilities near home was positively associated with physical activity in boys $(P R=$ 1.18; 95\%CI: 1.04-1.34). Among girls, distance ( $\geq$ 31 minutes) to sports courts was inversely associated with strength training $(P R=0.93 ; 95 \% C I$ : 0.87-0.99). The findings suggest that distance from home and number of recreational facilities in the neighborhood may affect patterns of physical activity among adolescents and that the association differs between boys and girls.

Motor Activity; Adolescent; Green Areas; Environment and Public Health
Alex Vieira Lima 1,2

Rogério César Fermino 1

Marcelo Ponestki Oliveira 1

Ciro Romelio Rodriguez Añez 3

Rodrigo Siqueira Reis 1,2

\section{Resumo}

O objetivo deste estudo foi verificar a associação entre a distância percebida até instalações de lazer (parques, praças, academias, ginásios e ciclovias) com a prática de atividade física e de exercícios em adolescentes de Curitiba, Paraná Brasil. Estudo transversal realizado com amostra representativa de 1.474 adolescentes (14-18 anos), alunos da rede pública de ensino. Foi verificada associação inversa entre a distância $\geq$ 31 minutos até academias e ginásios com a prática de atividade física nos meninos $(R P=0,78$; IC95\%: 0,69-0,88 e RP = 0,79; IC95\%: 0,66-0,95, respectivamente). O número de instalações próximas da residência associou-se positivamente com a prática de atividade física nos meninos ( $R P=1,18$; IC95\%: 1,04-1,34). Para as meninas, a distância $\geq 31$ minutos até um ginásio apresentou associação inversa com a prática de exercícios de força (RP = 0,93; IC95\%: 0,87-0,99). Esses achados sugerem que a distância e a quantidade de instalações de lazer no bairro podem afetar o padrão de atividade física de adolescentes, sendo essa relação diferente entre os sexos.

Atividade Motora; Adolescente; Áreas Verdes; Meio Ambiente e Saúde Pública 


\section{Introdução}

Os adolescentes têm adotado um estilo de vida fisicamente inativo, o que tem favorecido o aumento de diversas doenças crônicas não transmissíveis na idade adulta 1 . A prática regular de atividade física de intensidade moderada a vigorosa e exercícios de força e alongamento nessa faixa etária podem proporcionar benefícios para a saúde dos jovens, bem como a diminuição de fatores de risco para doenças cardiovasculares, além da melhora da saúde osteomioarticular e mental 2,3. Ainda, pesquisas mostram que adolescentes fisicamente ativos tendem a se tornar adultos igualmente ativos 4,5. No entanto, dados da Pesquisa Nacional de Saúde do Escolar (PeNSE) mostraram que mais da metade dos adolescentes brasileiros (57\%) não atinge as recomendações mínimas de prática de atividade física para a saúde 6 .

Evidências apontam que fatores individuais, interpessoais e ambientais estão associados com a prática de atividade física na adolescência 7,8,9. Dentre esses aspectos, acredita-se que as características do ambiente físico possam favorecer a prática de atividade física, com maior impacto para a saúde pública devido ao seu potencial em atingir um maior número de indivíduos 9,10. Aspectos como maior acessibilidade e menor percepção de distância até as instalações de lazer, maior densidade residencial, uso misto do solo, percepção de segurança e conectividade entre as ruas são atributos do ambiente, os quais apresentam consistente associação com a prática de atividade física de adolescentes 9,10. No entanto, essas evidências são limitadas a países de renda elevada, o que pode não representar as características de países de renda média como o Brasil 11.

No contexto brasileiro, estudos analisaram algumas características do ambiente como as barreiras percebidas para a prática de atividade física em adolescentes 12,13, porém não tiveram, como principal variável de exposição, os aspectos do ambiente como acesso, disponibilidade ou distância até as instalações de lazer. Por exemplo, Farias Júnior et al. 14 não observaram associação entre a percepção do ambiente com a prática de atividade física de intensidade moderada a vigorosa em adolescentes de João Pessoa, Paraíba. No entanto, Reis et al. 15 verificaram, em adolescentes de Curitiba, Paraná, que a percepção de proximidade até os parques está positivamente associada com prática de atividade física nesses locais. Porém, esse estudo 15 incluiu apenas características e prática de atividade física relacionadas aos parques da cidade. Estudos realizados em Curitiba, com emprego de medidas objetivas e subjetivas do ambiente, mostraram que maior acessibilidade até parques, ciclovias, pistas de caminhada e instalações de la- zer no bairro estão associadas com a prática de atividade física em adultos 16,17. Assim, acredita-se que a menor distância até diferentes espaços e estruturas recreativas possam favorecer a prática de atividade física de intensidade moderada a vigorosa e de exercícios de força e alongamento em adolescentes, o que pode ser um importante aspecto na compreensão da associação entre ambiente e atividade física 10 . Evidências sugerem que a prática de atividade física de lazer em adolescentes pode ser influenciada pela proximidade, disponibilidade e maior acesso a estruturas recreativas e equipamentos para a prática de atividade física no bairro ${ }^{10}$. A distância percebida é conceitualmente entendida como a percepção (portanto é uma medida subjetiva) do indivíduo sobre o tempo despendido de deslocamento até algum local para a prática de atividade física próximo de sua residência ${ }^{18}$. Estudos realizados em outros países mostraram associação positiva entre a proximidade até esses locais com a prática de atividade física em adolescentes 19,20.

Contudo, são inexistentes evidências sobre a associação entre a distância percebida até diferentes instalações de lazer com a prática de atividade física e de exercícios em adolescentes brasileiros. Ampliar essa compreensão é importante para a elaboração de estratégias e políticas de promoção da atividade física a partir das mudanças no ambiente ${ }^{21}$. Assim, o objetivo deste estudo foi verificar a associação entre a distância percebida e o número de instalações de lazer no bairro com a prática de atividade física de intensidade moderada a vigorosa e de exercícios em adolescentes de Curitiba.

\section{Materiais e métodos}

As informações utilizadas na presente pesquisa foram extraídas do banco de dados do projeto Determinantes da Atividade Física e Obesidade em Escolares do Ensino Médio da Rede Pública da Cidade de Curitiba, Paraná, Brasil. Em 2006, foi realizado um estudo transversal com amostra representativa de adolescentes que frequentavam o Ensino Médio, no período diurno, em escolas da rede pública de ensino de Curitiba ${ }^{22}$. De acordo com a Secretaria de Estado da Educação do Paraná (SEED), no ano de estudo, a população matriculada era de 42.563 escolares (SEED em números: relatório estatístico de alunos matriculados. http://wwwe.pr.gov.br/ escolas/numeros, acessado em 21/Set/2010).

\section{Participantes}

O cálculo amostral foi realizado considerando um erro de três pontos percentuais, prevalência estimada de $50 \%$ de inatividade física, efeito de deli- 
neamento de 1,5 , excesso de $10 \%$ para perdas e recusas e intervalo de 95\% de confiança (IC95\%) 22. Com base nesses dados, o tamanho da amostra foi estimado em 1.609 escolares 22. Para a estimativa do efeito do desenho, empregaram-se valores recomendados na literatura para pesquisas com estratégias de amostragens semelhantes às do presente estudo 23 .

A amostra foi selecionada, de maneira proporcional, por conglomerados, em dois estágios, considerando-se o número de alunos matriculados em cada uma das nove regionais da cidade. No primeiro estágio, foram sorteadas 18 escolas, a partir de uma relação fornecida pela SEED, de modo que a proporcionalidade fosse observada para cada regional. No segundo estágio, foram sorteadas 62 turmas, nas escolas previamente selecionadas, de maneira a atender a proporcionalidade entre a primeira, segunda e terceira séries do Ensino Médio, em cada uma das nove regionais.

\section{Coleta de dados}

A coleta de dados foi realizada de maneira coordenada, em sala de aula, com questionário padronizado, por dois entrevistadores previamente treinados. O estudo foi aprovado pelo Comitê de Ética em Pesquisa da Pontifícia Universidade Católica do Paraná (76/2006), e os procedimentos seguiram as recomendações do Sistema Nacional de Ética em Pesquisa.

\section{Variáveis dependentes}

\section{- Prática de atividade física de lazer}

A prática de atividade física, em uma semana habitual, foi avaliada com a seguinte questão 24 : "Considerando uma semana normal, em quantos dias você realizou atividades físicas por pelo menos 60 minutos por dia?". Os adolescentes foram orientados a considerar atividade física de lazer (sem considerar as aulas de Educação Física) como qualquer atividade de intensidade moderada a vigorosa (natação, jogos, dança, esportes, corridas, ginástica de academia etc.) que aumentasse a frequência cardíaca e respiratória ${ }^{25}$. As opções de resposta variaram de zero a sete dias por semana. Para efeito de análise, os participantes foram classificados como fisicamente ativos, segundo recomendações atuais de prática de atividade física para adolescentes 24 , de acordo com dois diferentes critérios: (a) praticar atividade física de intensidade moderada a vigorosa em pelo menos um dia na semana ( $\geq 1 \mathrm{vez} /$ semana, atividade física de intensidade moderada a vigorosa, $\geq 60 \mathrm{~min} /$ dia); e (b) praticar atividade física de intensidade moderada a vigorosa em pelo menos cinco dias na semana ( $\geq 5$ vezes/semana, atividade física de intensidade moderada a vigorosa, $\geq 60 \mathrm{~min} / \mathrm{dia}$ ) ${ }^{24}$.

\section{- Prática de exercícios de força}

A prática de exercícios de força foi avaliada com a seguinte questão: "Durante a última semana, em quantos dias você realizou exercícios de força (musculação, abdominais ou flexões)?”. A variável foi categorizada de acordo com as recomendações que sugerem, como adequada para a saúde osteomioarticular, a frequência semanal de prática igual ou superior a três vezes por semana ( $\geq 3$ vezes/ semana) 26,27 .

\section{- Prática de exercícios de alongamento}

A prática de exercícios de alongamento foi avaliada com a seguinte questão: "Na semana passada, em quantos dias você realizou exercícios de alongamento?". Sendo posteriormente categorizada segundo as evidências que recomendam a prática igual ou superior a três vezes por semana ( $\geq 3$ vezes/semana) 26,27.

\section{Variáveis independentes}

\section{- Distância percebida até as instalações de lazer}

A distância percebida até as instalações de lazer foi avaliada com uma questão previamente validada e utilizada em estudos similares $28,29,30$. Os adolescentes referiram o tempo, em minutos, que gastariam caminhando das suas residências até cada um dos seguintes locais mais próximos: parques, praças, academias de ginástica ou musculação, ginásios ou quadras para esportes e ciclovias ou pistas para caminhada. As opções de resposta apresentavam seis categorias: "1-5 min", "6-10 min", "11-20 min”, "21-30 min”, “+31 min” e "não sei”. As duas últimas categorias foram posteriormente agrupadas na análise. Essa classificação tem sido empregada em estudos similares, nos quais, a opção de resposta "não sei” é agrupada com a maior categoria de tempo (+31 min) 30 .

\section{- Número de instalações próximas da residência}

Evidências sugerem que a quantidade de atributos positivos do bairro está associada com a prática de atividade física em adultos 31, assim como o número de instalações de lazer próximas da residência pode favorecer a prática de atividade física em adultos 17 e adolescentes 32 . Por essa razão, optouse por criar uma variável que representa o número de instalações de lazer presentes em uma distân- 
cia de até 10 minutos de caminhada da residência, a qual foi categorizada em três níveis: 0 , entre 1-2 $\mathrm{e} \geq 3$ instalações.

\section{Covariáveis}

\section{- Faixa etária}

A faixa etária foi obtida com uma questão relacionada à idade, e os adolescentes foram classificados em cinco categorias: 14, 15, 16, 17 e 18 anos.

\section{- Índice de massa corporal}

O índice de massa corporal (IMC) foi obtido pela razão entre a massa corporal $(\mathrm{kg})$, dividida pela estatura elevada ao quadrado $\left(\mathrm{m}^{2}\right)$, a partir de medidas autorreferidas ${ }^{33}$. Para a classificação do IMC, foram empregados os pontos de corte sugeridos na literatura 34 , e os adolescentes foram classificados como "normal" (baixo peso e peso normal) ou "excesso de peso" (sobrepeso e/ou obesidade).

\section{- Nível econômico}

Para a classificação do nível econômico, foi utilizada a metodologia proposta pela Associação Brasileira de Empresas de Pesquisa (Critério de Classificação Econômica Brasil. http://www.abep.org, acessado em $20 / \mathrm{Fev} / 2008$ ), que avalia a presença de utensílios domésticos e a escolaridade do responsável financeiro pelo domicílio. Os adolescentes foram categorizados em três níveis: "A" (A1+A2 - maior renda), "B" (B1+B2) e "C" $(\mathrm{C}+\mathrm{D}+\mathrm{E})$.

\section{- Percepção de saúde}

A percepção de saúde foi avaliada com a questão 35 "De modo geral, você diria que a sua saúde é": as opções de resposta apresentavam escala tipo Likert de quatro pontos ("ruim", "regular", "boa" e "excelente”). As respostas "ruim" e "regular", assim como as opções "boa" e "excelente", foram agrupadas nas análises para caracterizar as categorias de percepção de saúde negativa e positiva, respectivamente.

\section{Análise dos dados}

Entre os 1.518 adolescentes entrevistados, 44 não apresentavam dados completos nas variáveis utilizadas neste estudo e foram excluídos das análises restando 1.474 participantes. Os dados foram analisados pela frequência absoluta e relativa, comparando-se as proporções de prática de atividade física e de exercícios de força e alongamento entre as categorias de distância percebida até as instalações de lazer. A regressão de Poisson foi utilizada para analisar a associação entre as variáveis. Após a elaboração do modelo bruto, todas as variáveis independentes e as covariáveis foram inseridas no mesmo nível de análise pelo método de entrada forçada para a elaboração do modelo múltiplo. As análises foram realizadas no programa Stata 11 (Stata Corp., College Station, Estados Unidos), e o processo de amostragem por conglomerados foi considerado recorrendo-se ao comando svy do software. As análises foram estratificadas por sexo, e o nível de significância, mantido em $5 \%$.

\section{Resultados}

A amostra analítica foi constituída por 1.474 adolescentes (59\% do sexo feminino), e o percentual de recusas para a participação na pesquisa foi inferior a $5 \%(n=79)$. Cerca de um terço dos adolescentes (33\%) encontrava-se na faixa etária de 16 anos, a maioria apresentou IMC normal $(88,1 \%)$, nível econômico intermediário (B - 62,6\%) e percepção positiva de saúde $(86,4 \%)$. Essas variáveis diferiram entre os sexos $(\mathrm{p}<0,05)$, e ainda a maior faixa etária, o excesso de peso, a renda mais elevada e a percepção positiva de saúde foram associados com o sexo masculino (Tabela 1). Aproximadamente, seis a cada dez adolescentes praticavam atividade física de intensidade moderada a vigorosa $\geq 1 \mathrm{vez} /$ semana $(57,9 \%)$, enquanto que $14,3 \%$ praticavam atividade física de intensidade moderada a vigorosa $\geq 5$ vezes/semana. Os meninos foram fisicamente mais ativos em ambas as categorias analisadas (atividade física de intensidade moderada a vigorosa $\geq 1 \mathrm{vez} /$ semana: $75 \%$ vs. $46,2 \%$; atividade física de intensidade moderada a vigorosa $\geq 5$ vezes/semana: $21,7 \%$ vs. $9,2 \%$; $\mathrm{p}<$ 0,001). A prática recomendada de exercícios de força e alongamento também foi reportada com maior frequência pelos meninos $(40,3 \% v s .17,2 \%$ e $57,2 \%$ vs. $45,8 \%$, respectivamente; $\mathrm{p}<0,001$ ). As praças foram as instalações reportadas como mais próximas da residência ( $<5$ min: $33,6 \%$ ), enquanto os parques foram os locais mais distantes $(\geq 31$ min: $50,5 \%)$. As meninas perceberam maior distância até as instalações de lazer $(\mathrm{p}<0,05)$. Aproximadamente, quatro a cada dez adolescentes reportaram a presença de uma ou duas instalações próximas da residência, e as meninas relataram inexistência de instalações mais frequentemente do que os meninos ( $21,4 \%$ vs. $13,3 \%$; $\mathrm{p}<0,001)$. Os resultados relativos a essas variáveis estão descritos, por sexo, na Tabela 1.

A Tabela 2 apresenta os resultados da análise bivariada. Para as meninas, as distâncias de 6-10 minutos e $\geq 31$ minutos até uma praça foram inversamente associadas com a prática de atividade física de intensidade moderada a vigorosa $\geq 1$ vez/ 
Tabela 1

Características sociodemográficas, prática de atividade física e de exercícios e distância percebida até as instalações de lazer entre os participantes. Curitiba, Paraná, Brasil, 2006.

\begin{tabular}{|c|c|c|c|c|c|c|c|}
\hline \multirow[t]{2}{*}{ Variável } & \multicolumn{2}{|c|}{ Meninos $(n=603)$} & \multicolumn{2}{|c|}{ Meninas $(n=871)$} & \multirow{2}{*}{$\begin{array}{l}\text { Valor } \\
\text { de } p\end{array}$} & \multicolumn{2}{|c|}{ Todos $(\mathrm{N}=1.474)$} \\
\hline & $n$ & $\%$ & $\mathrm{n}$ & $\%$ & & $\mathrm{n}$ & $\%$ \\
\hline \multicolumn{8}{|l|}{ Faixa etária (anos) } \\
\hline 14 & 30 & 5,0 & 56 & 6,4 & 0,041 & 86 & 5,8 \\
\hline 15 & 138 & 22,9 & 221 & 25,4 & & 359 & 24,4 \\
\hline 16 & 198 & 32,8 & 289 & 33,2 & & 487 & 33,0 \\
\hline 17 & 181 & 30,0 & 238 & 27,3 & & 419 & 28,4 \\
\hline 18 & 56 & 9,3 & 67 & 7,7 & & 123 & 8,3 \\
\hline \multicolumn{8}{|c|}{ Índice de massa corporal } \\
\hline Normal & 503 & 83,4 & 796 & 91,4 & $<0,001$ & 1.299 & 88,1 \\
\hline Excesso de peso & 100 & 16,6 & 75 & 8,6 & & 175 & 11,9 \\
\hline \multicolumn{8}{|l|}{ Nível econômico } \\
\hline A (maior renda) & 129 & 21,4 & 145 & 16,6 & 0,004 & 274 & 18,6 \\
\hline B & 378 & 62,7 & 545 & 62,6 & & 923 & 62,6 \\
\hline C & 96 & 15,9 & 181 & 20,8 & & 277 & 18,8 \\
\hline \multicolumn{8}{|l|}{ Percepção de saúde } \\
\hline Negativa & 57 & 9,5 & 144 & 16,5 & $<0,001$ & 201 & 13,6 \\
\hline Positiva & 546 & 90,5 & 727 & 83,5 & & 1.273 & 86,4 \\
\hline \multicolumn{8}{|c|}{ Prática de atividade física de intensidade moderada a } \\
\hline \multicolumn{8}{|c|}{ vigorosa (vezes/semana) } \\
\hline 0 & 151 & 25,0 & 469 & 53,8 & $<0,001$ & 620 & 42,1 \\
\hline$\geq 1$ & 452 & 75,0 & 402 & 46,2 & & 854 & 57,9 \\
\hline$\leq 4$ & 472 & 78,3 & 791 & 90,8 & $<0,001$ & 1.263 & 85,7 \\
\hline$\geq 5$ & 131 & 21,7 & 80 & 9,2 & & 211 & 14,3 \\
\hline \multicolumn{8}{|c|}{ Prática de exercícios de força (vezes/semana) } \\
\hline$\leq 2$ & 360 & 59,7 & 721 & 82,8 & $<0,001$ & 1.081 & 73,3 \\
\hline$\geq 3$ & 243 & 40,3 & 150 & 17,2 & & 393 & 26,7 \\
\hline \multicolumn{8}{|c|}{ Prática de exercícios de alongamento (vezes/semana) } \\
\hline$\leq 2$ & 258 & 42,8 & 472 & 54,2 & $<0,001$ & 730 & 49,5 \\
\hline$\geq 3$ & 345 & 57,2 & 399 & 45,8 & & 744 & 50,5 \\
\hline \multicolumn{8}{|c|}{ Distância percebida até as instalações de lazer } \\
\hline \multicolumn{8}{|l|}{ Parques (minutos) } \\
\hline$<5$ & 66 & 10,9 & 84 & 9,6 & 0,013 & 150 & 10,2 \\
\hline $6-10$ & 81 & 13,4 & 87 & 10,0 & & 168 & 11,4 \\
\hline $11-20$ & 95 & 15,8 & 123 & 14,1 & & 218 & 14,8 \\
\hline $21-30$ & 78 & 12,9 & 115 & 13,2 & & 193 & 13,1 \\
\hline$\geq 31$ & 283 & 46,9 & 462 & 53,0 & & 745 & 50,5 \\
\hline \multicolumn{8}{|l|}{ Praças (minutos) } \\
\hline$<5$ & 234 & 38,8 & 262 & 30,1 & $<0,001$ & 496 & 33,6 \\
\hline $6-10$ & 120 & 19,9 & 164 & 18,8 & & 284 & 19,3 \\
\hline $11-20$ & 86 & 14,3 & 151 & 17,3 & & 237 & 16,1 \\
\hline $21-30$ & 49 & 8,1 & 65 & 7,5 & & 114 & 7,7 \\
\hline$\geq 31$ & 114 & 18,9 & 229 & 26,3 & & 343 & 23,3 \\
\hline
\end{tabular}




\begin{tabular}{|c|c|c|c|c|c|c|c|}
\hline \multirow[t]{2}{*}{ Variável } & \multicolumn{2}{|c|}{ Meninos $(n=603)$} & \multicolumn{2}{|c|}{ Meninas $(n=871)$} & \multirow{2}{*}{$\begin{array}{l}\text { Valor } \\
\text { de } p\end{array}$} & \multicolumn{2}{|c|}{ Todos $(\mathrm{N}=1.474$} \\
\hline & n & $\%$ & $\mathrm{n}$ & $\%$ & & $\mathrm{n}$ & $\%$ \\
\hline \multicolumn{8}{|c|}{ Distância percebida até as instalações de lazer } \\
\hline \multicolumn{8}{|c|}{ Academias de ginástica/musculação (minutos) } \\
\hline$<5$ & 137 & 22,7 & 159 & 18,3 & 0,016 & 296 & 20,1 \\
\hline $6-10$ & 144 & 23,9 & 194 & 22,3 & & 338 & 22,9 \\
\hline $11-20$ & 129 & 21,4 & 197 & 22,6 & & 326 & 22,1 \\
\hline $21-30$ & 67 & 11,1 & 113 & 13,0 & & 180 & 12,2 \\
\hline$\geq 31$ & 126 & 20,9 & 208 & 23,9 & & 334 & 22,7 \\
\hline \multicolumn{8}{|c|}{ Ginásios ou quadras para esportes (minutos) } \\
\hline$<5$ & 165 & 27,4 & 141 & 16,2 & $<0,001$ & 306 & 20,8 \\
\hline $6-10$ & 142 & 23,5 & 160 & 18,4 & & 302 & 20,5 \\
\hline $11-20$ & 110 & 18,2 & 164 & 18,8 & & 274 & 18,6 \\
\hline $21-30$ & 75 & 12,4 & 93 & 10,7 & & 168 & 11,4 \\
\hline$\geq 31$ & 111 & 18,4 & 313 & 35,9 & & 424 & 28,8 \\
\hline \multicolumn{8}{|c|}{ Ciclovias ou pistas para caminhada (minutos) } \\
\hline$<5$ & 188 & 31,2 & 221 & 25,4 & 0,001 & 409 & 27,7 \\
\hline $6-10$ & 103 & 17,1 & 146 & 16,8 & & 249 & 16,9 \\
\hline $11-20$ & 87 & 14,4 & 110 & 12,6 & & 197 & 13,4 \\
\hline $21-30$ & 47 & 7,8 & 63 & 7,3 & & 110 & 7,5 \\
\hline$\geq 31$ & 178 & 29,5 & 331 & 38,0 & & 509 & 34,5 \\
\hline \multicolumn{8}{|c|}{ Número de instalações de lazer próximas da residência * } \\
\hline 0 & 80 & 13,3 & 186 & 21,4 & $<0,001$ & 266 & 18,1 \\
\hline $1-2$ & 257 & 42,6 & 389 & 44,7 & & 646 & 43,8 \\
\hline$\geq 3$ & 266 & 44,1 & 296 & 34,0 & & 562 & 38,1 \\
\hline
\end{tabular}

* Até 10 minutos de caminhada.

semana $(R P=0,80$; IC95\%: 0,69-0,93 e RP = 0,77; IC95\%: 0,59-0,99, respectivamente). Entre os meninos, a distância $\geq 31$ minutos até uma academia de ginástica foi inversamente associada com a prática de atividade física de intensidade moderada a vigorosa $\geq 1 \mathrm{vez} /$ semana $(\mathrm{RP}=0,76$; IC95\%: 0,660,88 ), a distância de 21-30min até um ginásio apresentou associação positiva com a prática de exercício de alongamento ( $\mathrm{RP}=1,08$; IC95\%: 1,0-1,16), enquanto a distância $\geq 31$ min foi inversamente associada com a prática de atividade física de intensidade moderada a vigorosa $\geq 1 \mathrm{vez} /$ semana ( $\mathrm{RP}=0,77$; IC95\%: 0,64-0,91). Também, a distância de 11-20 min até uma ciclovia ou pista para caminhada foi inversamente associada com a prática de atividade física de intensidade moderada a vigorosa $\geq 5 \mathrm{vez} / \mathrm{semana}(\mathrm{RP}=0,51$; IC95\%: 0,27-0,97) e de exercícios de força ( $R P=0,89$; IC95\%: 0,80-0,99). Por fim, a presença de três ou mais instalações de lazer próximas da residência associou-se positivamente com a prática de atividade física de intensidade moderada a vigorosa $\geq 1 \mathrm{vez} /$ semana $(\mathrm{RP}=$ 1,20; IC95\%: 1,05-1,36).

Os resultados da análise multivariável são apresentados na Tabela 3. Para as meninas, a per- cepção de distância entre 6-10 min até uma praça permaneceu inversamente associada com a prática de atividade física de intensidade moderada a vigorosa $\geq 1 \mathrm{vez} / \mathrm{semana}(\mathrm{RP}=0,77$; IC95\%: 0,67 0,88 ). Para os meninos, a distância $\geq 31$ min até uma academia de ginástica ou musculação $(\mathrm{RP}=$ 0,78; IC95\%: 0,69-0,88) e ginásio ou quadras para esportes (RP = 0,79; IC95\%: 0,66-0,95) manteve-se inversamente associada com a prática de atividade física de intensidade moderada a vigorosa $\geq 1$ vez/semana. Também para as meninas, a distância $\geq 31$ min até um ginásio apresentou associação inversa com a prática de exercícios de força $(\mathrm{RP}=$ 0,93; IC95\%: 0,87-0,99). A distância percebida de 11-20 min até uma ciclovia ou pistas para caminhada manteve a associação inversa com a prática de exercícios de força para os meninos $(\mathrm{RP}=0,88$; IC95\%: 0,80-0,96). Por fim, a presença de três ou mais instalações de lazer próximas da residência manteve-se positivamente associada com a prática de atividade física de intensidade moderada a vigorosa $\geq 1 \mathrm{vez} /$ semana para os meninos ( $\mathrm{RP}=$ 1,18; IC95\%: 1,04-1,34)

Foi verificada tendência de associação positiva entre o número de instalações de lazer próximas 
Tabela 2

Associação bivariada entre a distância percebida até as instalações de lazer com a prática de atividade física e de exercícios em adolescentes. Curitiba, Paraná, Brasil, $2006(N=1.474)$.

\begin{tabular}{|c|c|c|c|c|c|c|c|c|}
\hline \multirow[t]{3}{*}{ Distância } & \multicolumn{8}{|c|}{ Meninos $(n=603)$} \\
\hline & \multicolumn{2}{|c|}{$\begin{array}{c}\text { Atividade física de } \\
\text { intensidade moderada } \\
\text { a vigorosa } \\
\text { ( } \geq 1 \text { vez/semana) }\end{array}$} & \multicolumn{2}{|c|}{$\begin{array}{c}\text { Atividade física de } \\
\text { intensidade moderada } \\
\text { a vigorosa } \\
\text { ( } \geq 5 \text { vezes/semana) }\end{array}$} & \multicolumn{2}{|c|}{$\begin{array}{l}\text { Exercícios de força } \\
\text { ( }(3 \text { vezes/semana) }\end{array}$} & \multicolumn{2}{|c|}{$\begin{array}{c}\text { Exercícios de } \\
\text { alongamento } \\
\text { ( } 3 \text { vezes/semana) }\end{array}$} \\
\hline & $\mathrm{RP}$ & IC95\% & $\mathrm{RP}$ & IC95\% & $\mathrm{RP}$ & IC95\% & RP & IC95\% \\
\hline \multicolumn{9}{|c|}{ Parques (minutos) } \\
\hline$<5$ & 1,00 & & 1,00 & & 1,00 & & 1,00 & \\
\hline $6-10$ & 1,04 & $0,82-1,32$ & 0,91 & $0,49-1,70$ & 1,06 & $0,97-1,16$ & 1,02 & $0,90-1,15$ \\
\hline $11-20$ & 0,96 & $0,81-1,13$ & 0,73 & $0,42-1,26$ & 0,96 & $0,88-1,04$ & 0,94 & $0,85-1,04$ \\
\hline $21-30$ & 0,89 & $0,74-1,09$ & 0,89 & $0,42-1,90$ & 1,06 & $0,93-1,19$ & 1,01 & $0,86-1,20$ \\
\hline$\geq 31$ & 1,04 & $0,89-1,22$ & 0,91 & $0,57-1,46$ & 1,01 & $0,93-1,10$ & 1,02 & $0,93-1,11$ \\
\hline \multicolumn{9}{|c|}{ Praças (minutos) } \\
\hline$<5$ & 1,00 & & 1,00 & & 1,00 & & 1,00 & \\
\hline $6-10$ & 0,99 & $0,90-1,09$ & 0,87 & $0,56-1.36$ & 1,01 & $0,94-1,08$ & 0,99 & $0,88-1,11$ \\
\hline $11-20$ & 1,03 & $0,91-1,18$ & 1,06 & $0,66-1,72$ & 0,96 & $0,90-1,03$ & 1,01 & $0,94-1,09$ \\
\hline $21-30$ & 0,91 & $0,74-1,08$ & 1,03 & $0,63-1,67$ & 0,93 & $0,83-1,05$ & 1,04 & $0,92-1,17$ \\
\hline$\geq 31$ & 0,93 & $0,82-1,04$ & 1,04 & $0,58-1,86$ & 0,99 & $0,88-1,11$ & 1,00 & $0,92-1,09$ \\
\hline \multicolumn{9}{|c|}{$\begin{array}{l}\text { Academias de ginástica ou } \\
\text { musculação (minutos) }\end{array}$} \\
\hline$<5$ & 1,00 & & 1,00 & & 1,00 & & 1,00 & \\
\hline $6-10$ & 1,01 & $0,90-1,13$ & 0,89 & $0,60-1,34$ & 1,00 & $0,94-1,08$ & 0,99 & $0,90-1,09$ \\
\hline $11-20$ & 0,96 & $0,85-1,08$ & 0,61 & $0,36-1,06$ & 0,98 & $0,90-1,06$ & 1,00 & $0,92-1,08$ \\
\hline $21-30$ & 0,95 & $0,80-1,13$ & 0,90 & $0,48-1,69$ & 1,01 & $0,92-1,11$ & 0,97 & $0,84-1,11$ \\
\hline$\geq 31$ & 0,76 & $0,66-0,88$ & 0,73 & $0,44-1,18$ & 0,93 & $0,86-1,00$ & 0,93 & $0,81-1,06$ \\
\hline \multicolumn{9}{|c|}{ Ginásios ou quadras para esportes } \\
\hline \multicolumn{9}{|l|}{ (minutos) } \\
\hline$<5$ & 1,00 & & 1,00 & & 1,00 & & 1,00 & \\
\hline $6-10$ & 1,01 & $0,95-1,08$ & 0,96 & $0,62-1,50$ & 0,96 & $0,90-1,04$ & 0,95 & $0,87-1,07$ \\
\hline $11-20$ & 0,98 & $0,88-1,10$ & 0,92 & $0,65-1,31$ & 1,01 & $0,92-1,09$ & 0,96 & $0,88-1,03$ \\
\hline $21-30$ & 0,98 & $0,85-1,15$ & 0,64 & $0,38-1,03$ & 1,04 & $0,93-1,16$ & 1,08 & $1,01-1,16$ \\
\hline$\geq 31$ & 0,77 & $0,64-0,91$ & 0,56 & $0,31-1,01$ & 0,89 & $0,79-1,00$ & 0,94 & $0,87-1,00$ \\
\hline \multirow{2}{*}{\multicolumn{9}{|c|}{$\begin{array}{l}\text { Ciclovias ou pistas para caminhada } \\
\text { (minutos) }\end{array}$}} \\
\hline & & & & & & & & \\
\hline$<5$ & 1,00 & & 1,00 & & 1,00 & & 1,00 & \\
\hline $6-10$ & 1,06 & $0,98-1,15$ & 0,87 & $0,54-1,40$ & 0,97 & $0,86-1,09$ & 1,00 & $0,93-1,07$ \\
\hline $11-20$ & 0,88 & $0,76-1,01$ & 0,51 & $0,27-0,97$ & 0,89 & $0,80-0,99$ & 0,91 & $0,82-1,02$ \\
\hline $21-30$ & 0,89 & $0,76-1,05$ & 0,88 & $0,48-1,59$ & 0,95 & $0,85-1,05$ & 1,03 & $0,95-1,12$ \\
\hline$\geq 31$ & 0,90 & $0,80-1,01$ & 0,71 & $0,51-1,00$ & 0,96 & $0,87-1,05$ & 0,96 & $0,89-1,04$ \\
\hline \multicolumn{9}{|c|}{ Número de instalações de lazer } \\
\hline \multicolumn{9}{|c|}{ próximas da residência * } \\
\hline 0 & 1,00 & & 1,00 & & 1,00 & & 1,00 & \\
\hline $1-2$ & 1,09 & $0,91-1,31$ & 1,37 & $0,62-3,00$ & 1,02 & $0,92-1,14$ & 1,01 & $0,93-1,11$ \\
\hline$\geq 3$ & 1,20 & $1,05-1,36$ & 1,65 & $0,83-3,26$ & 1,08 & $0,93-1,24$ & 1,01 & $0,92-1,11$ \\
\hline
\end{tabular}

(continua) 
Tabela 2 (continuação)

\begin{tabular}{|c|c|c|c|c|c|c|c|c|}
\hline \multirow[t]{3}{*}{ Distância } & \multicolumn{8}{|c|}{ Meninas $(n=871)$} \\
\hline & \multicolumn{2}{|c|}{$\begin{array}{c}\text { Atividade física de } \\
\text { intensidade moderada } \\
\text { a vigorosa } \\
\text { ( } \geq 1 \text { vez/semana) }\end{array}$} & \multicolumn{2}{|c|}{$\begin{array}{c}\text { Atividade física de } \\
\text { intensidade moderada } \\
\text { a vigorosa } \\
\text { ( } \geq 5 \text { vezes/semana) }\end{array}$} & \multicolumn{2}{|c|}{$\begin{array}{l}\text { Exercícios de força } \\
\text { ( } \geq 3 \text { vezes/semana) }\end{array}$} & \multicolumn{2}{|c|}{$\begin{array}{c}\text { Exercícios de } \\
\text { alongamento } \\
(\geq 3 \text { vezes/semana) }\end{array}$} \\
\hline & RP & IC95\% & $\mathrm{RP}$ & IC95\% & RP & IC95\% & RP & IC95\% \\
\hline \multicolumn{9}{|c|}{ Parques (minutos) } \\
\hline$<5$ & 1,00 & & 1,00 & & 1,00 & & 1,00 & \\
\hline $6-10$ & 0,98 & $0,67-1,44$ & 0,78 & $0,31-2,00$ & 1,02 & $0,95-1,10$ & 1,08 & $0,93-1,25$ \\
\hline $11-20$ & 0,91 & $0,58-1,42$ & 0,55 & $0,12-2,46$ & 0,96 & $0,90-1,02$ & 1,05 & $0,95-1,17$ \\
\hline $21-30$ & 0,86 & $0,65-1,15$ & 0,73 & $0,26-1,98$ & 0,95 & $0,88-1,03$ & 1,07 & $0,97-1,17$ \\
\hline$\geq 31$ & 0,91 & $0,69-1,20$ & 0,66 & $0,34-1,27$ & 0,97 & $0,92-1,01$ & 1,02 & $0,92-1,14$ \\
\hline \multicolumn{9}{|c|}{ Praças (minutos) } \\
\hline$<5$ & 1,00 & & 1,00 & & 1,00 & & 1,00 & \\
\hline $6-10$ & 0,80 & $0,69-0,93$ & 1,15 & $0,81-1,63$ & 0,95 & $0,88-1,02$ & 0,98 & $0,92-1,04$ \\
\hline $11-20$ & 1,12 & $0,95-1,34$ & 1,24 & $0,72-2,16$ & 1,03 & $0,94-1,12$ & 0,98 & $0,93-1,02$ \\
\hline $21-30$ & 1,15 & $0,89-1,49$ & 0,78 & $0,32-1,93$ & 1,00 & $0,89-1,11$ & 1,03 & $0,97-1,09$ \\
\hline$\geq 31$ & 0,77 & $0,59-0,99$ & 0,64 & $0,27-1,50$ & 0,97 & $0,90-1,04$ & 0,96 & $0,87-1,06$ \\
\hline \multicolumn{9}{|c|}{ Academias de ginástica ou } \\
\hline \multicolumn{9}{|c|}{ musculação (minutos) } \\
\hline$<5$ & 1,00 & & 1,00 & & 1,00 & & 1,00 & \\
\hline $6-10$ & 1,17 & $0,93-1,46$ & 0,72 & $0,28-1,87$ & 1,02 & $0,93-1,12$ & 1,04 & $0,95-1,15$ \\
\hline $11-20$ & 1,04 & $0,88-1,24$ & 0,71 & $0,40-1,28$ & 1,03 & $0,97-1,08$ & 1,01 & $0,92-1,11$ \\
\hline $21-30$ & 1,12 & $0,82-1,53$ & 1,01 & $0,41-2,46$ & 1,02 & $0,96-1,09$ & 1,02 & $0,95-1,19$ \\
\hline$\geq 31$ & 0,92 & $0,73-1,17$ & 0,72 & $0,37-1,38$ & 0,98 & $0,89-1,09$ & 0,94 & $0,85-1,03$ \\
\hline \multirow{2}{*}{\multicolumn{9}{|c|}{$\begin{array}{l}\text { Ginásios ou quadras para esportes } \\
\text { (minutos) }\end{array}$}} \\
\hline & & & & & & & & \\
\hline$<5$ & 1,00 & & 1,00 & & 1,00 & & 1,00 & \\
\hline $6-10$ & 0,90 & $0,64-1,27$ & 0,67 & $0,28-1,61$ & 0,92 & $0,85-1,00$ & 0,97 & $0,87-1,19$ \\
\hline $11-20$ & 0,88 & $0,67-1,15$ & 0,80 & $0,40-1,63$ & 0,97 & $0,91-1,04$ & 0,98 & $0,89-1,07$ \\
\hline $21-30$ & 1,00 & $0,77-1,29$ & 0,89 & $0,53-1,48$ & 0,97 & $0,88-1,06$ & 1,01 & $0,92-1,09$ \\
\hline$\geq 31$ & 0,77 & $0,59-1,01$ & 0,63 & $0,34-1,18$ & 0,93 & $0,87-1,00$ & 0,95 & $0,88-1,02$ \\
\hline \multirow{2}{*}{\multicolumn{9}{|c|}{$\begin{array}{l}\text { Ciclovia ou pistas para caminhada } \\
\text { (minutos) }\end{array}$}} \\
\hline & & & & & & & & \\
\hline$<5$ & 1,00 & & 1,00 & & 1,00 & & 1,00 & \\
\hline $6-10$ & 0,84 & $0,67-1,03$ & 0,66 & $0,33-1,32$ & 1,00 & $0,90-1,11$ & 0,99 & $0,93-1,07$ \\
\hline $11-20$ & 1,07 & $0,86-1,34$ & 0,88 & $0,46-1,68$ & 1,02 & $0,94-1,09$ & 1,02 & $0,95-1,09$ \\
\hline $21-30$ & 1,13 & $0,97-1,32$ & 0,70 & $0,23-2,05$ & 1,00 & $0,94-1,07$ & 1,03 & $0,94-1,14$ \\
\hline$\geq 31$ & 0,87 & $0,69-1,09$ & 0,74 & $0,46-1,19$ & 1,00 & $0,96-1,03$ & 0,97 & $0,92-1,01$ \\
\hline \multicolumn{9}{|c|}{ Número de instalações de lazer } \\
\hline \multicolumn{9}{|c|}{ próximas da residência * } \\
\hline 0 & 1,00 & & 1,00 & & 1,00 & & 1,00 & \\
\hline $1-2$ & 0,89 & $0,71-1,10$ & 1,25 & $0,74-2,09$ & 1,01 & $0,93-1,10$ & 1,00 & $0,95-1,05$ \\
\hline$\geq 3$ & 1,03 & $0,82-1,30$ & 1,59 & $0,85-2,98$ & 1,03 & $0,95-1,11$ & 1,04 & $0,97-1,11$ \\
\hline
\end{tabular}

IC95\%: intervalo de 95\% de confiança; RP: razão de prevalência.

* Até 10 minutos de caminhada. 
Tabela 3

Associação multivariável * entre a distância percebida até as instalações de lazer com a prática de atividade física e de exercícios em adolescentes. Curitiba, Paraná, Brasil ( $\mathrm{N}=1.474)$.

\begin{tabular}{|c|c|c|c|c|c|c|c|c|}
\hline \multirow[t]{3}{*}{ Distância } & \multicolumn{8}{|c|}{ Meninos ( $n=603$ ) } \\
\hline & \multicolumn{2}{|c|}{$\begin{array}{c}\text { Atividade física de } \\
\text { intensidade moderada } \\
\text { a vigorosa } \\
\text { ( } \geq 1 \text { vez/semana) }\end{array}$} & \multicolumn{2}{|c|}{$\begin{array}{c}\text { Atividade física de } \\
\text { intensidade moderada } \\
\text { a vigorosa } \\
\text { ( } 25 \text { vezes/semana) }\end{array}$} & \multicolumn{2}{|c|}{$\begin{array}{l}\text { Exercícios de força } \\
\text { ( } 23 \text { vezes/semana) }\end{array}$} & \multicolumn{2}{|c|}{$\begin{array}{c}\text { Exercícios de } \\
\text { alongamento } \\
(\geq 3 \text { vezes/semana) }\end{array}$} \\
\hline & $\mathrm{RP}$ & IC95\% & $\mathrm{RP}$ & IC95\% & $\mathrm{RP}$ & IC95\% & $\mathrm{RP}$ & IC95\% \\
\hline \multicolumn{9}{|c|}{ Parques (minutos) } \\
\hline$<5$ & 1,00 & & 1,00 & & 1,00 & & 1,00 & \\
\hline $6-10$ & 1,05 & $0,86-1,29$ & 0,92 & $0,50-1,68$ & 1,06 & $0,96-1,18$ & 1,01 & $0,90-1,12$ \\
\hline $11-20$ & 0,98 & $0,86-1,17$ & 0,76 & $0,40-1,45$ & 0,96 & $0,88-1,04$ & 0,94 & $0,86-1,03$ \\
\hline $21-30$ & 0,94 & $0,83-1,13$ & 1,01 & $0,45-2,24$ & 1,05 & $0,91-1,22$ & 1,03 & $0,87-1,21$ \\
\hline$\geq 31$ & 1,05 & $0,92-1,20$ & 0,97 & $0,61-1,54$ & 1,02 & $0,93-1,12$ & 1,03 & $0,95-1,11$ \\
\hline \multicolumn{9}{|c|}{ Praças (minutos) } \\
\hline$<5$ & 1,00 & & 1,00 & & 1,00 & & 1,00 & \\
\hline $6-10$ & 0,99 & $0,89-1,11$ & 0,88 & $0,57-1,36$ & 1,02 & $0,95-1,10$ & 0,98 & $0,89-1,09$ \\
\hline $11-20$ & 1,01 & $0,93-1,08$ & 1,07 & $0,67-1,69$ & 0,98 & $0,91-1,06$ & 1,01 & $0,94-1,08$ \\
\hline $21-30$ & 0,89 & $0,74-1,08$ & 1,05 & $0,65-1,69$ & 0,94 & $0,85-1,03$ & 1,03 & $0,92-1,16$ \\
\hline$\geq 31$ & 0,93 & $0,82-1,05$ & 1,19 & $0,70-2,04$ & 1,02 & $0,92-1,13$ & 1,02 & $0,93-1,10$ \\
\hline \multicolumn{9}{|c|}{$\begin{array}{l}\text { Academias de ginástica ou } \\
\text { musculação (minutos) }\end{array}$} \\
\hline$<5$ & 1,00 & & 1,00 & & 1,00 & & 1,00 & \\
\hline $6-10$ & 1,03 & $0,93-1,13$ & 0,87 & $0,58-1,30$ & 1,01 & $0,94-1,07$ & 0,99 & $0,91-1,08$ \\
\hline $11-20$ & 1,02 & $0,89-1,17$ & 0,67 & $0,40-1,10$ & 0,99 & $0,92-1,07$ & 1,01 & $0,93-1,10$ \\
\hline $21-30$ & 0,96 & $0,81-1,15$ & 0,92 & $0,50-1,71$ & 1,03 & $0,95-1,12$ & 0,97 & $0,85-1,10$ \\
\hline$\geq 31$ & 0,78 & $0,69-0,88$ & 0,82 & $0,50-1,32$ & 0,97 & $0,89-1,05$ & 0,95 & $0,83-1,08$ \\
\hline \multicolumn{9}{|c|}{ Ginásios ou quadras para esportes } \\
\hline \multicolumn{9}{|l|}{ (minutos) } \\
\hline$<5$ & 1,00 & & 1,00 & & 1,00 & & 1,00 & \\
\hline $6-10$ & 0,99 & $0,91-1,07$ & 0,93 & $0,59-1,46$ & 0,97 & $0,90-1,05$ & 0,95 & $0,87-1,04$ \\
\hline $11-20$ & 0,98 & $0,86-1,11$ & 0,93 & $0,67-1,28$ & 1,00 & $0,93-1,08$ & 0,95 & $0,88-1,03$ \\
\hline $21-30$ & 1,01 & $0,87-1,18$ & 0,62 & $0,36-1,06$ & 1,04 & $0,93-1,15$ & 1,08 & $1,00-1,16$ \\
\hline$\geq 31$ & 0,79 & $0,66-0,95$ & 0,64 & $0,34-1,19$ & 0,91 & $0,83-1,01$ & 0,94 & $0,87-1,02$ \\
\hline \multicolumn{9}{|c|}{ Ciclovias ou pistas para } \\
\hline \multicolumn{9}{|c|}{ caminhada (minutos) } \\
\hline$<5$ & 1,00 & & 1,00 & & 1,00 & & 1,00 & \\
\hline $6-10$ & 1,09 & $0,98-1,21$ & 0,89 & $0,58-1,38$ & 0,98 & $0,87-1,09$ & 0,99 & $0,93-1,06$ \\
\hline $11-20$ & 0,95 & $0,80-1,12$ & 0,55 & $0,30-1,00$ & 0,88 & $0,80-0,96$ & 0,91 & $0,82-1,01$ \\
\hline $21-30$ & 0,93 & $0,80-1,07$ & 0,93 & $0,51-1,71$ & 0,95 & $0,85-1,06$ & 1,03 & $0,94-1,13$ \\
\hline$\geq 31$ & 0,92 & $0,82-1,04$ & 0,78 & $0,56-1,07$ & 0,98 & $0,90-1,06$ & 0,97 & $0,91-1,03$ \\
\hline \multirow{2}{*}{\multicolumn{9}{|c|}{$\begin{array}{l}\text { Número de instalações de lazer } \\
\text { próximas da residência ** }\end{array}$}} \\
\hline & & & & & & & & \\
\hline 0 & 1,00 & & 1,00 & & 1,00 & & 1,00 & \\
\hline $1-2$ & 1,09 & $0,91-1,30$ & 1,33 & $0,60-2,92$ & 1,01 & $0,89-1,14$ & 1,00 & $0,92-1,10$ \\
\hline$\geq 3$ & 1,18 & $1,04-1,34$ & 1,47 & $0,74-2,91$ & 1,04 & $0,91-1,20$ & 1,00 & $0,91-1,09$ \\
\hline
\end{tabular}




\begin{tabular}{|c|c|c|c|c|c|c|c|c|}
\hline \multirow[t]{3}{*}{ Distância } & \multicolumn{8}{|c|}{ Meninas $(n=871)$} \\
\hline & \multicolumn{2}{|c|}{$\begin{array}{c}\text { Atividade física de } \\
\text { intensidade moderada } \\
\text { a vigorosa } \\
\text { ( } \geq 1 \text { vez/semana) }\end{array}$} & \multicolumn{2}{|c|}{$\begin{array}{c}\text { Atividade física de } \\
\text { intensidade moderada } \\
\text { a vigorosa } \\
\text { ( } \geq 5 \text { vezes/semana) }\end{array}$} & \multicolumn{2}{|c|}{$\begin{array}{l}\text { Exercícios de força } \\
\text { ( } \geq 3 \text { vezes/semana) }\end{array}$} & \multicolumn{2}{|c|}{$\begin{array}{c}\text { Exercícios de } \\
\text { alongamento } \\
\text { ( } 23 \text { vezes/semana) }\end{array}$} \\
\hline & RP & IC95\% & RP & IC95\% & RP & IC95\% & RP & IC95\% \\
\hline \multicolumn{9}{|c|}{ Parques (minutos) } \\
\hline$<5$ & 1,00 & & 1,00 & & 1,00 & & 1,00 & \\
\hline $6-10$ & 1,01 & $0,75-1,35$ & 0,78 & $0,32-1,86$ & 1,02 & $0,96-1,10$ & 1,08 & $0,93-1,25$ \\
\hline $11-20$ & 0,96 & $0,66-1,39$ & 0,55 & $0,12-2,42$ & 0,95 & $0,90-1,01$ & 1,06 & $0,94-1,19$ \\
\hline $21-30$ & 0,89 & $0,68-1,16$ & 0,72 & $0,27-1,90$ & 0,95 & $0,88-1,02$ & 1,07 & $0,97-1,18$ \\
\hline$\geq 31$ & 0,94 & $0,75-1,18$ & 0,67 & $0,37-1,19$ & 0,97 & $0,92-1,02$ & 1,03 & $0,92-1,14$ \\
\hline \multicolumn{9}{|c|}{ Praças (minutos) } \\
\hline$<5$ & 1,00 & & 1,00 & & 1,00 & & 1,00 & \\
\hline $6-10$ & 0,77 & $0,67-0,88$ & 1,23 & $0,86-1,73$ & 0,96 & $0,90-1,04$ & 0,99 & $0,93-1,05$ \\
\hline $11-20$ & 1,09 & $0,93-1,26$ & 1,22 & $0,72-2,12$ & 1,03 & $0,95-1,11$ & 0,97 & $1,93-1,01$ \\
\hline $21-30$ & 1,17 & $0,93-1,49$ & 0,83 & $0,35-1,96$ & 0,98 & $0,88-1,10$ & 1,01 & $0,95-1,07$ \\
\hline$\geq 31$ & 0,81 & $0,65-1,01$ & 0,70 & $0,29-1,67$ & 0,98 & $0,91-1,05$ & 0,98 & $0,89-1,07$ \\
\hline \multicolumn{9}{|c|}{$\begin{array}{l}\text { Academias de ginástica ou } \\
\text { musculação (minutos) }\end{array}$} \\
\hline$<5$ & 1,00 & & 1,00 & & 1,00 & & 1,00 & \\
\hline $6-10$ & 1,21 & $1,03-1,42$ & 0,77 & $0,29-2,02$ & 1,03 & $0,93-1,13$ & 1,04 & $0,95-1,15$ \\
\hline $11-20$ & 1,09 & $0,96-1,23$ & 0,73 & $1,40-1,31$ & 1,03 & $0,98-1,08$ & 1,02 & $0,93-1,12$ \\
\hline $21-30$ & 1,12 & $0,88-1,41$ & 1,03 & $0,44-2,38$ & 1,03 & $0,97-1,10$ & 1,04 & $0,97-1,11$ \\
\hline$\geq 31$ & 0,95 & $0,80-1,13$ & 0,73 & $0,36-1,45$ & 0,99 & $0,90-1,08$ & 0,95 & $0,87-1,04$ \\
\hline \multicolumn{9}{|c|}{$\begin{array}{l}\text { Ginásios ou quadras para esportes } \\
\text { (minutos) }\end{array}$} \\
\hline$<5$ & 1,00 & & 1,00 & & 1,00 & & 1,00 & \\
\hline $6-10$ & 0,94 & $0,72-1,22$ & 0,68 & $0,29-1,60$ & 0,92 & $0,85-1,00$ & 0,91 & $0,85-1,09$ \\
\hline $11-20$ & 0,90 & $0,70-1,16$ & 0,82 & $0,41-1,66$ & 0,99 & $0,92-1,06$ & 0,98 & $0,89-1,08$ \\
\hline $21-30$ & 1,00 & $0,78-1,30$ & 0,88 & $0,55-1,41$ & 0,96 & $0,88-1,06$ & 0,99 & $0,90-1,09$ \\
\hline$\geq 31$ & 0,81 & $0,61-1,06$ & 0,61 & $0,34-1,08$ & 0,93 & $0,87-0,99$ & 0,94 & $0,86-1,01$ \\
\hline \multicolumn{9}{|c|}{$\begin{array}{l}\text { Ciclovias ou pistas para } \\
\text { caminhada (minutos) }\end{array}$} \\
\hline$<5$ & 1,00 & & 1,00 & & 1,00 & & 1,00 & \\
\hline $6-10$ & 0,89 & $0,74-1,09$ & 0,68 & $0,34-1,37$ & 1,00 & $0,90-1,11$ & 1,00 & $0,93-1,07$ \\
\hline $11-20$ & 1,10 & $0,91-1,33$ & 0,89 & $0,44-1,77$ & 1,01 & $0,94-1,08$ & 1,02 & $0,96-1,08$ \\
\hline $21-30$ & 1,23 & $0,99-1,53$ & 0,75 & $0,24-2,35$ & 1,00 & $0,93-1,08$ & 1,03 & $0,95-1,13$ \\
\hline$\geq 31$ & 0,91 & $0,74-1,11$ & 0,77 & $0,50-1,19$ & 1,00 & $0,97-1,03$ & 0,98 & $0,94-1,02$ \\
\hline \multicolumn{9}{|c|}{ Número de instalações de lazer } \\
\hline \multicolumn{9}{|c|}{ próximas da residência ** } \\
\hline 0 & 1,00 & & 1,00 & & 1,00 & & 1,00 & \\
\hline $1-2$ & 0,85 & $0,68-1,05$ & 1,28 & $0,79-2,07$ & 1,02 & $0,94-1,11$ & 1,01 & $0,95-1,06$ \\
\hline$\geq 3$ & 0,97 & $0,76-1,23$ & 1,59 & $0,84-3,00$ & 1,03 & $0,95-1,11$ & 1,04 & $0,97-1,11$ \\
\hline
\end{tabular}

IC95\%: intervalo de 95\% de confiança; RP: razão de prevalência.

* Ajustado para faixa etária, IMC (índice de massa corporal), nível econômico e percepção de saúde;

** Até 10 minutos de caminhada. 
da residência com a frequência de prática de atividade física de intensidade moderada a vigorosa e de exercícios de força (Figura 1).

\section{Discussão}

Este é o primeiro estudo que analisa a associação entre a distância percebida até as instalações de lazer com a prática de atividade física de intensidade moderada a vigorosa e de exercícios de força e alongamento em amostra representativa de adolescentes brasileiros. As evidências são consistentes e demonstram que a maior proximidade e o acesso a diferentes espaços e estruturas recreativas para prática de atividade física estão positivamente associados com a prática de atividade física em adolescentes de países de renda elevada 9,10,15,30,32,36. Ao contrário do esperado, não foi verificada associação positiva entre a proximidade da residência até parques, praças, academias, ginásios e ciclovias com a prática de atividade física de intensidade moderada a vigorosa e de exercícios na maior parte das análises realizadas.

No presente estudo, apenas os meninos que relataram residir a uma distância superior a 31 min até academias de ginástica ou ginásios apresentaram menor probabilidade de praticar atividade física de intensidade moderada a vigorosa $\geq 1 \mathrm{vez} / \mathrm{semana}$. Esse resultado pode ser explicado pelo elevado tempo para se deslocar até esses locais ser percebido como barreira e dificultar o engajamento nas atividades. De fato, as principais barreiras para a prática de atividade física relatadas por adolescentes de Curitiba incluem a percepção de distância até os locais, dificuldade de acesso e não conhecer os locais, além da falta de segurança no bairro 12,13. De maneira similar ao verificado no presente estudo, em pesquisas conduzidas no Brasil (Curitiba) 15, na Holanda (Roterdã) 19, em Portugal (Porto) 37 e na Nova Zelândia (Auckland) 20, os autores encontraram associação positiva entre presença e proximidade de instalações de lazer (parques, quadras, academias e ciclovias) com a prática de atividade física de adolescentes. Contudo, esses resultados são distintos aos encontrados em João Pessoa, onde a percepção de presença de locais para a prática de atividade física no bairro não se associou com a prática de atividade física de intensidade moderada a vigorosa 14. Essa parcial inconsistência pode ser explicada por diferenças metodológicas na medida do ambiente entre as pesquisas (distâncias até os locais $v s$. presença de locais).

O número de instalações de lazer próximas da residência foi positivamente associada com a prática de atividade física de intensidade moderada a vigorosa $\geq 1 \mathrm{vez} / \mathrm{semana}$ entre os meninos. Esses

\section{Figura 1}

Associação entre o número de instalações de lazer próximas da residência com a frequência de prática de atividade física e de exercícios em adolescentes. Curitiba, Paraná, Brasil, 2006 ( $\mathrm{N}=1.474)$.

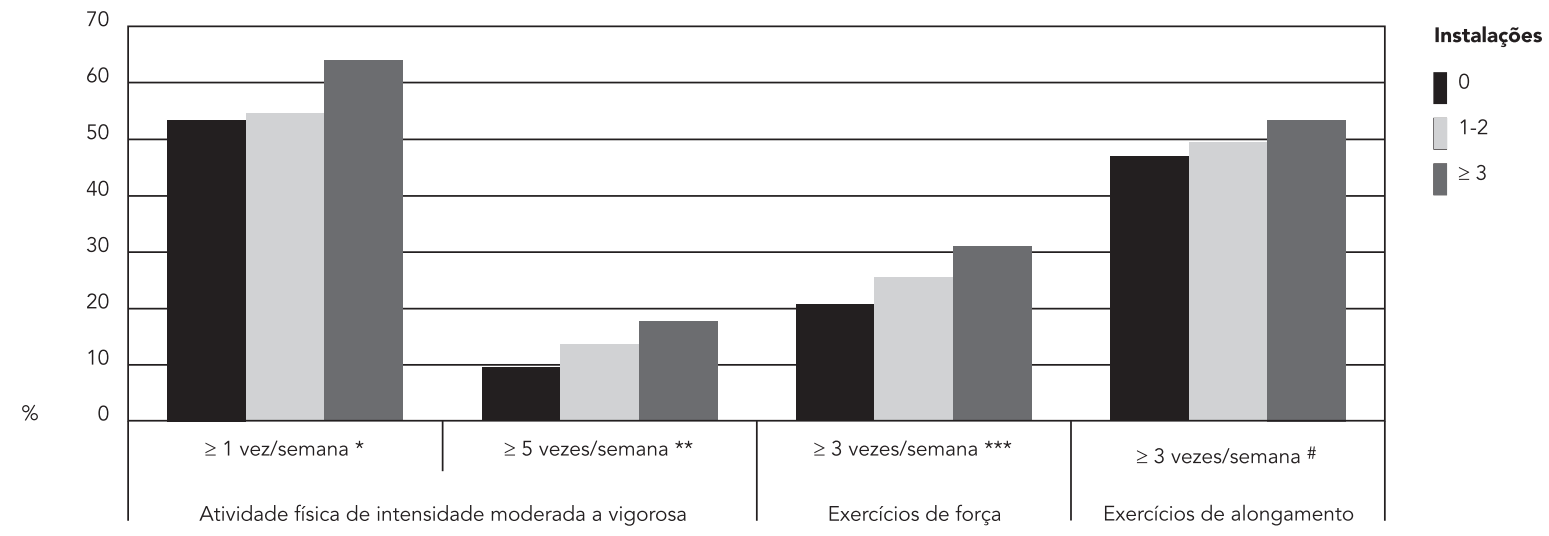

$$
\begin{aligned}
& { }^{\star} p=0,001 \\
& { }^{\star \star} p=0,001 \\
& { }^{\star \star *} p=0,001 \\
& \# p=0,065
\end{aligned}
$$


resultados são similares aos encontrados em pesquisas conduzidas nos Estados Unidos 32,38 , em Portugal ${ }^{39}$, na Austrália 40 e na China ${ }^{41}$. De fato, a maior densidade e variedade de instalações de lazer próximas da residência podem favorecer a prática de atividade física de intensidade moderada a vigorosa. Evidências apontam que os adolescentes praticam atividade física de intensidade moderada a vigorosa, principalmente, nos finais de semana 42 , e que as atividades mais comuns são modalidades esportivas como futebol, vôlei, basquete, handebol, entre outros 43 . Algumas das instalações de lazer avaliadas no presente estudo (parques, praças, ginásios e quadras) apresentam diversas estruturas para a prática das modalidades supracitadas 44 , as quais poderiam facilitar a realização das atividades durante o tempo de lazer, como os finais de semana.

Entre as meninas, a menor distância percebida até as praças foi inversamente associada com a prática de atividade física de intensidade moderada a vigorosa $\geq 1 \mathrm{vez} / \mathrm{semana}$. As meninas percebem um maior número de barreiras para a prática de atividade física, as quais estão relacionadas com aspectos motivacionais (baixa autoeficácia, preguiça, preferência por outras atividades) e sociais (apoio social em geral, ocupação, falta de tempo) 12,13. A elevada permanência em atividades domésticas, além da falta de apoio social da família para a prática de atividade física em locais próximos à residência podem dificultar o envolvimento em atividade física 43 . Adicionalmente, a percepção de insegurança no bairro e a dificuldade de acesso até os locais, entre outros, estão inversamente associadas com a prática de atividade física em parques de Curitiba 15,45. É possível que essas características dificultem a prática de atividade física, independente da proximidade das instalações de lazer presentes no bairro.

Os resultados sobre a associação entre a distância percebida e a disponibilidade de instalações de lazer com a prática exercícios de força e alongamento foram inconsistentes. Por exemplo, enquanto, entre os meninos, verificou-se associação inversa entre a percepção de distância de 11-20 min até uma ciclovia com a prática de exercícios de força; entre as meninas, essa associação foi observada com a distância percebida $\geq 31 \mathrm{~min}$ até um ginásio. A inexistência de evidências que tenham analisado essa associação limita a comparação dos resultados. Porém, aspectos como a presença e a qualidade dos equipamentos nesses locais não foram avaliadas, e essas características poderiam explicar a associação entre proximidade dos locais com a realização de exercícios ${ }^{15}$. Além disso, alguns locais analisados podem não oferecer estruturas adequadas para a prática de atividades físicas preferidas pelos adolescentes. Por exemplo, em Curitiba, alguns parques e praças contam com aparelhos para a prática de exercícios de força e alongamento 15 , porém a literatura reporta que os adolescentes preferem atividades físicas menos estruturadas (esportes em geral) 46 .

Algumas limitações devem ser consideradas ao interpretar os resultados. O delineamento transversal empregado não permite determinar a direção das associações. Porém, foram analisados desfechos múltiplos de atividade física, e os principais fatores de confusão foram controlados. As instalações de lazer analisadas não contemplam todas as possibilidades de espaços e estruturas para a prática de atividade física dos adolescentes. A faixa etária dos adolescentes foi limitada entre 14 a 18 anos de idade, matriculados na rede pública de Curitiba, o que limita a extrapolação dos resultados para escolares que estudam em escolas privadas. Por outro lado, os procedimentos utilizados para a seleção da amostra possibilitaram a redução dos vieses de seleção, e o tamanho amostral foi suficiente para detectar os efeitos nas análises multivariadas.

\section{Conclusão}

A percepção de longas distâncias até as instalações de lazer foi inversamente associada com a prática de atividade física em meninos, enquanto que o número de instalações apresentou associação positiva para esse desfecho. Para as meninas, a maior distância até as instalações foi inversamente associada com a prática de exercícios de força. A distância e a quantidade de instalações de lazer podem afetar o padrão de prática de atividade física e de exercícios em adolescentes, mas esse comportamento difere entre os sexos. Diminuir as distâncias e aumentar o número de instalações de lazer facilitaria o comportamento ativo dos adolescentes. Esses achados possibilitam que os gestores públicos elaborem intervenções baseadas em modificações do ambiente construído, o que inclui a construção e a manutenção de instalações de lazer para a promoção da atividade física na população. Sugere-se que futuros estudos avaliem os efeitos das modificações ambientais na comunidade, as quais devem facilitar o acesso a diversas estruturas, sobre o nível de atividade física de adolescentes. 


\section{Resumen}

El objetivo de este estudio fue investigar la asociación entre la distancia percibida a instalaciones recreativas (parques, gimnasios, gimnasios y carriles-bici) para la práctica de actividad física y el ejercicio en los adolescentes de Curitiba, Paraná, Brasil. Es un estudio transversal con una muestra representativa de 1.474 adolescentes (14-18 años), estudiantes de escuelas públicas. Se observó una asociación inversa entre la distancia $\geq 31$ minutos hasta los gimnasios y centros de acondicionamiento físico con la práctica de la actividad física de los niños $(R P=0,78$; IC95\%: 0,69-0,88 y RP = 0,79; IC95\%: 0,66-0,95, respectivamente). El número de lugares cerca de la residencia se asoció positivamente con la práctica de la actividad física de los niños (RP = 1,18; IC95\%: 1,04-1,34). Para las niñas, la distancia $\geq 31$ minutos a un gimnasio se asoció inversamente con el ejercicio del poder ( $R P=0$, 93; IC95\%: 0,87-0,99). Estos hallazgos sugieren que la distancia y la cantidad de instalaciones de ocio en la zona puede afectar el patrón de actividad física de los adolescentes, diferenciándose esta relación entre sexos.

Actividad Motora; Adolescente; Áreas Verdes; Medio Ambiente y Salud Pública

\section{Colaboradores}

A. V. Lima participou da concepção do estudo, revisão da literatura, análise dos dados e redação inicial do manuscrito. R. C. Fermino colaborou na concepção inicial do estudo, revisão da literatura, análise dos dados, redação e revisão crítica do manuscrito em todas as suas etapas. M. P. Oliveira contribuiu na concepção do artigo. C. R. Rodriguez Añez e R. S. Reis coordenaram a coleta de dados e participaram da revisão crítica do texto.

\section{Referências}

1. Lee IM, Shiroma EJ, Lobelo F, Puska P, Blair SN, Katzmarzyk PT. Effect of physical inactivity on major non-communicable diseases worldwide: an analysis of burden of disease and life expectancy. Lancet 2012; 380:219-29.

2. Hallal PC, Victora CG, Azevedo MR, Wells JCK. Adolescent physical activity and health: a systematic review. Sports Med 2006; 36:1019-30.

3. Andersen LB, Harro M, Sardinha LB, Froberg K, Ekelund U, Brage S. Physical activity and clustered cardiovascular risk in children: a cross-sectional study (the European youth heart study). Lancet 2006; 368:299-304.

4. Azevedo MR, Araújo CL, Cozzenza SM, Hallal PC. Tracking of physical activity from adolescent to adukthood: a population-based study. Rev Saúde Pública 2007; 41:69-75.

5. Raustorp A, Ekroth Y. Tracking of pedometer determined physical activity: a 10 years follow-up study from adolescence to adulthood in Sweden. J Phys Act Health 2013; [Epub ahead of print].
6. Hallal PC, Knuth AG, Cruz DKA, Mendes MI, Malta D. Prática de atividade física em adolescentes brasileiros. Ciênc Saúde Coletiva 2010; 15:3035-42.

7. Sallis JF, Prochaska JJ, Taylor WC. A review of correlates of physical activity of children and adolescents. Med Sci Sports Exerc 2000; 32:963-75.

8. Van der Horst K, Oenema A, Ferreira I, Wendel-Vos W, Giskes K, van Lenthe F, et al. A systematic review of environmental correlates of obesity-related dietary behaviors in youth. Health Educ Res 2007; 22:203-26.

9. Bauman AB, Reis RS, Sallis JF, Wells JC, Loos RJF, Martin BW. Correlates of physical activity: why are some people physically active and others not? Lancet 2012; 380:35-44.

10. Ding D, Sallis JF, Kerr J, Lee S, Rosenberg DE. Neighborhood environment and physical activity among youth a review. Am J Prev Med 2011; 41:442-55 
11. Rydin Y, Bleahu A, Davies M, Dávila JD, Friel S, Grandis GD, et al. Shaping cities for health: complexity and the planning of urban environments in the 21st century. Lancet 2012; 379:2079-108.

12. Santos MS, Fermino RC, Reis RS, Cassou AC, Anez CR. Barriers related to physical activity practice in adolescents. a focus-group study. Rev Bras Cineantropom Desempenho Hum 2010;12:137-43.

13. Santos MS, Hino AA, Reis RS, Anez CR. Prevalence of barriers for physical activity in adolescents. Rev Bras Epidemiol 2010; 13:94-104.

14. Farias Júnior JC, Lopes AS, Mota J, Santos MP, Ribeiro JC, Hallal PC. Perception if the social and buith environment and physical activity among Northeastern Brazil adolescents. Prev Med 2011; 52:114-9.

15. Reis RS, Hino AA, Florindo AA, Anez CR, Domingues MR. Association between physical activity in parks and perceived environment: a study with adolescents. J Phys Act Health 2009; 6:503-9.

16. Hino AA, Reis RS, Sarmiento OL, Parra DC, Brownson RC. The built environment and recreational physical activity among adults in Curitiba, Brazil. Prev Med 2011; 52:419-22.

17. Parra DC, Hoehner CM, Hallal PC, Ribeiro IC, Reis RS, Brownson RC, et al. Perceived environmen tal correlates of physical activity for leisure and transportation in Curitiba, Brazil. Prev Med 2011 52:234-8

18. Kahn EB, Ramsey LT, Brownson RC, Heath GW, Howze EH, Powell KE, et al. The effectiveness of in terventions to increase physical activity. Am J Prev Med 2002; 4:77-107.

19. Prins RG, Oenema A, van der Horst K, Brug J. Objective and perceived availability of physical activity opportunities: differences in associations with physical activity behavior among urban adolescents. Int J Behav Nutr Phys Act 2009; 6:70.

20. Utter J, Denny S, Robinson EM, Ameratunga S, Watson P. Perceived access to community facilities, social motivation, and physical activity among New Zealand youth. J Adolesc Health 2006; 39:770-3.

21. Reis RS, Kelly CM, Parra DC, Barros M, Gomes G, Malta D, et al. Developing a research agenda for promoting physical activity in Brazil through environmental and policy change. Rev Panam Salud Pública 2012; 32:93-100.

22. Fermino RC, Rech CR, Hino AAF, Rodriguez Añez C, Reis RS. Atividade física e fatores associados em adolescentes do ensino médio de Curitiba, Brasil. Rev Saúde Pública 2010; 44:986-95.

23. Luiz RR, Magnanini MMF. A lógica da determinação do tamanho da amostra em investigações epidemiológicas. Cad Saúde Colet (Rio J.) 2000; 8:9-28.

24. Brener ND, Kann L, Kinchen SA, Grunbaum JA, Whalen L, Eaton D. Methodology of the youth risk behavior surveillance system. MMWR Recomm Rep 2004; 53:1-13.

25. Hallal PC, Gomez LF, Parra DC, Lobelo F, Mosquera J, Florindo AA, et al. Lessons learned after 10 years of IPAQ use in Brazil and Colombia. J Phys Act Health 2010; 7 Suppl 2:S259-64.

26. Strong WB, Malina RM, Blimkie CJ, Daniels SR, Dishman RK, Gutin B. Evidence based physical activity for school-age youth. J Pediatr 2005; 146:732-7.
27. U.S. Department of Health and Human Services Physical activity guidelines advisory committee. Washington DC: U.S. Department of Health and Human Services; 2008.

28. Saelens BE, Sallis JF, Black JB, Chen D. Neighborhood-based differences in physical activity: an environment scale evaluation. Am J Public Health 2003; 93:1552-8.

29. Rosenberg D, Ding D, Sallis JF, Kerr J, Norman GJ, Durant N, et al. Neighborhood Environment Walkability Scale for Youth (NEWS-Y): reliability and relationship with physical activity. Prev Med 2009; 49:213-8

30. Grow HM, Saelens BE, Kerr J, Durant NH, Norman GJ, Sallis JF. Where are youth active? roles of proximity, active transport, and built environment. Med Sci Sports Exerc 2008; 40:71-9.

31. Sallis JF, Bowles HR, Bauman A, Ainsworth BE, Bull FC, Craig CL, et al. Neighborhood environments and physical activity among adults in 11 countries. Am J Prev Med 2009; 36:484-90.

32. Frank L, Kerr J, Chapman J, Sallis J. Urban form relationships with walk trip frequency and distance among youth. Am J Health Promot 2007; 21:305-11.

33. Farias Junior JC. Validade das medidas autorreferi das de peso e estatura para o diagnóstico do estado nutricional de adolescentes. Rev Bras Saúde Matern Infant 2007; 7:167-74

34. Cole TJ, Bellizzi MC, Flegal KM, DietzWH. Establishing a standard definition for child overweight and obesity worldwide: international survey. BMJ 2000; 320:1240-3

35. Piko BF. Self-perceived health among adolescents: the role of gender and psychosocial factors. Eur J Pediatr 2007; 166:701-8.

36. Roemmich JN, Epstein LH, Raja S, Yin L, Robinson J, Winiewicz D. Association of access to parks and recreational facilities with the physical activity of young children. Prev Med 2006;43:437-41.

37. Mota J, Almeida M, Santos R, Ribeiro JC, Santos MP. Association of perceived environmental characteristics and participation in organized and nonorganized physical activities of adolescents. Pediatr Exerc Sci 2009; 21:233-9.

38. Gordon-Larsen P, Nelson MC, Page P, Popkin BM. Inequality in the built environment underlies key health disparities in physical activity and obesity. Pediatrics 2006; 117:417-24.

39. Mota J, Almeida M, Santos P, Ribeiro JC. Perceived neighborhood environments and physical activity in adolescents. Prev Med 2005; 41:834-6.

40. Timperio A, Giles-Corti B, Crawford D. Features of public open spaces and physical activity among children: findings from the CLAN study. Prev Med 2008; 47:514-8

41. Li M, Dibley M, Sibbritt D, Yan H. Factors associated with adolescents' physical inactivity in Xi'an City, China. Med Sci Sports Exerc 2006; 38:2075-85.

42. Lima RBCP, Fermino RC, Seabra A, Garganta R, Maia JAR. Padrão de atividade física em crianças e jovens: um breve resumo do estado do conhecimento. Rev Bras Cineantropom Desempenho Hum 2010; 12:68-76. 
43. Gonçalves H, Hallal PC, Amorim TC, Araújo CLP, Menezes AMB. Fatores socioculturais e nível de atividade física no início da adolescência. Rev Panam Salud Pública 2007; 22:246-53.

44. Hino AAF, Reis RS, Ribeiro IC, Parra DC, Brownson RC, Fermino RC. Using observational methods to evaluate public open spaces and physical activity in Brazil. J Phys Act Health 2010;7 Suppl 2:S146-54.

45. Price G, Reis RS. Making kid-friendly cities: lessons from two cities. Prev Med 2010; 50 Suppl 1:S95-6.
46. Hallal PC, Bertoldi AD, Gonçalves H, Victora CG. Prevalência de sedentarismo e fatores associados em adolescentes de 10-12 anos de idade. Cad Saúde Pública 2006; 22:1277-87.

Recebido em 10/Dez/2012

Versão final reapresentada em 06/Mar/2013

Aprovado em 21/Mar/2013 